\title{
Reform Of The Computer Network Training Courses
}

\author{
Liang Wang \\ Science and Technology Research Department \\ Shanghai Urban Management College, Shanghai China 200232 \\ E-Mail:civilwon@hotmail.com
}

Keywords: Computer; Network Education; courses; Lan

\begin{abstract}
Ccomputer network technology training allows students to master the conventional mode of operation in the whole process of curriculum really.Understanding of the theory, students after training, can learn to network technical knowledge and methods of operation can be directly applied to the day-to-day work in the future, and able to grasp the correct way to learn through training to enable them to lifelong. This paper will build the significance of the system of the computer network training courses, courses required for the network environment to build, how to build a network curriculum system point of view of network training curriculum reform thinking.
\end{abstract}

\section{The role of the computer network training courses}

Computer network technology is one of the computer network professional core courses, teaches students the network operating system and network device management knowledge. The current higher education, often emphasis on theory-style education, and because a relatively small number of hours of the course and content of the coverage is relatively narrow, give students hands-on course provides too few opportunities, and thus only through this course of learning, mastery of the knowledge of the network may not be solid, easily lead to the students' first post forget. And computer network technology in the teaching process, often encounter the following situation:

\subsection{The purely theoretical teaching}

Currently, many colleges and universities in the computer network technology course using the traditional lecture-style teaching methods, rather than the students directly involved, the whole process of teaching basic teachers as principal, and students only "audience", which ignores the teaching and learning synchronization and fundamental, the students in the classroom can not be the actual operation. Which one could have in the classroom will be hands-on and brains combination of courses become dull, unable to stimulate students' interest in network.

\subsection{Lack of appropriate network laboratory equipment}

Computer Network Technology is a very practical course, in the experiment not only do software experiment hardware experiment has to be done, which requires specialized computer network training room. Learning network technology If you do not do the experiment is equal to the paper, such as network protocols, LAN configuration, forgot without hands tend to learn first. At present, many vocational colleges do not have a dedicated computer network training room, shared with other courses ordinary computer lab, thus leading to many teachers under conditions of limited experimental arrangements only arrangements based experiments, and for many experimental settings and adjustment of computer network security, the settings of the LAN, switches, routers, servers, etc.

\section{Computer network training environment construction}

The training room requires the best use of transparent glass-style anti-static flooring static floor, this floor so that students can visually see the entire training room network network cabling design, considerable knowledge of integrated wiring for the students to masterhelp. 
VMware software installed in your computer's operating system and run multiple operating systems at the same time. Such as the installation of Windows Server 2008 computer with VMWARE install and run a Windows XP and Windows 7, so Windows Server 2008, Windows XP and Windows 7 on one computer to form a local area network, so that it can communicate with each other and can help each other access shared files, as shown in Figure 1.

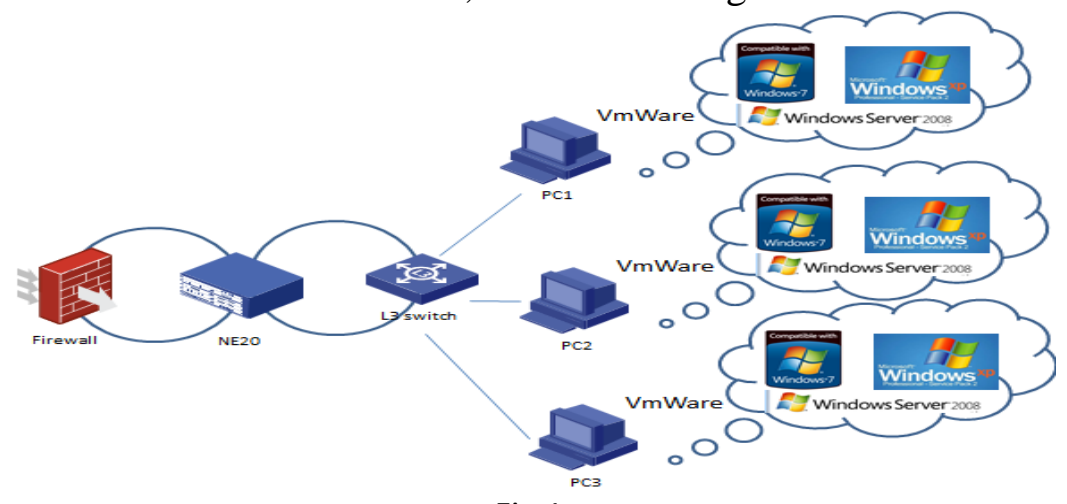

Fig 1

\section{Computer network training course content}

The entire computer network training course consists of five parts: computer assembly, LAN architecture design and management of Windows Server 2008 network operating system, Linux operating system management, cabling infrastructure, routers, switches, configuration and management.

\subsection{Computer Assembly}

As a qualified computer administrator, master configure and assemble a cost-effective, high-quality computer skills necessary to master these premises must have a rich knowledge of hardware, a variety of products of the brand models, parameters and other details to be very understanding. The same time, the computer software functionality and compatibility, and other issues, but also need to have rich knowledge of the software and software applications, hardware and software so as to achieve a reasonable match to assemble a high-performance and cost-effective computer.

\subsection{Windows Server 2008 LAN architecture design and management}

Windows Server 2008 is the current mainstream network operating system, many of the company's servers using the operating system to provide day-to-day network access and data management. Therefore, Windows Server 2008 learning throughout the training course is particularly important. The training includes the use of the Microsoft Management Console, domain creation, use and management of domain user accounts and groups to create and manage object permissions management, group policy, and audit policy management, server performance monitoring, IP address, and The VLSM divided and management. Master design and management of the network architecture, which includes create and manage DHCP server, DNS configuration and management, the creation of the IIS server and management knowledge.

\subsection{Linux operating system management}

Computer software applications is a very practical skills, so direct practical experience of the users often play a irreplaceable role. Based server applications in many companies in addition to Microsoft Windows Server platform, as well as much of the open-source Linux platform, but Linux is divided into multiple software versions depending on the application. Enterprise applications based on the current status quo, and a lot of real network environment using Red Hat Linux version 
of this course is to select Red Hat Enterprise Linux Server 6.0 version (hereinafter referred RHELS6.0). For most students, it may be the first contact with Linux, so many students are unfamiliar interface of the operating system, while the majority of the operation command is also relatively unknown, as shown in Figure 2.

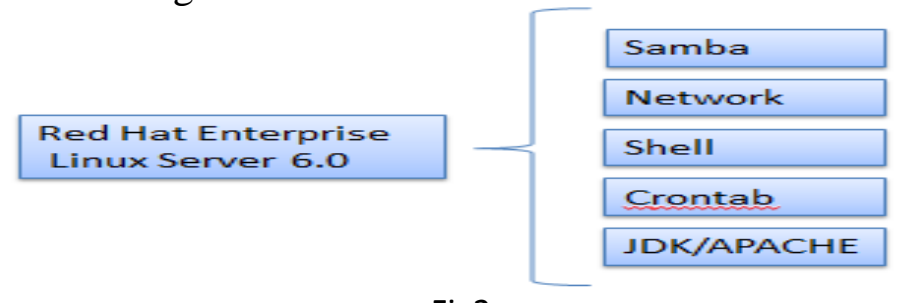

Fig2

\subsection{1 the Linux foundation knowledge}

Tells the origin of the Linux, Linux system installation method, the Linux command line using Linux, Linux graphical interface to use, in the common application of third party software. By mastering the operating system is the most basic knowledge, let the students know what is Linux, Linux has what effect, the Linux installation way and method, can grasp the basic software applications at the same time, learning to lay a solid foundation for subsequent chapters.

\subsubsection{Linux system administration}

Linux system administration including the localization of the Linux operating system management, including user and group management concepts and practical application, and file systems, Linux system performance tuning and monitoring system, system configuration and common management maintenance methods, etc. Based on the learning of knowledge points, and through the corresponding operation training, to master basic knowledge management of commonly used Linux systems.

\subsubsection{Linux network management}

Linux network configuration management consists of a network, the basic knowledge of different network configuration services is the most important. For students with a higher vocational computer major, must master the Linux network configuration of the DNS, DHCP services, WEB services, E-mail services, NFS services. By studying these configuration method, the Linux system configured to DNS, DHCP, the WEB server.

\subsection{Integrated wiring}

Integrated wiring system is a technology for voice, data, video and other information of the standard of structured cabling system, as a transmission network, it can make the voice and data communications devices are connected, and can make the network switching equipment and other information management systems connected to each other. At present many enterprise network administrators to exclude both conventional network software failure, and to solve the network hardware failure, so for the network hardware wiring design and management of learning is particularly important.

\subsection{Router and switch management}

With increasing development of network technology, network faster and faster, data processing capabilities network devices are becoming increasingly powerful. As a network administrator, addition day-to-day operating system and application software maintenance, but also need to master device configuration management network hardware, network through combination software and hardware management in order to maintain normal, healthy operation. Therefore, the day-to-day 


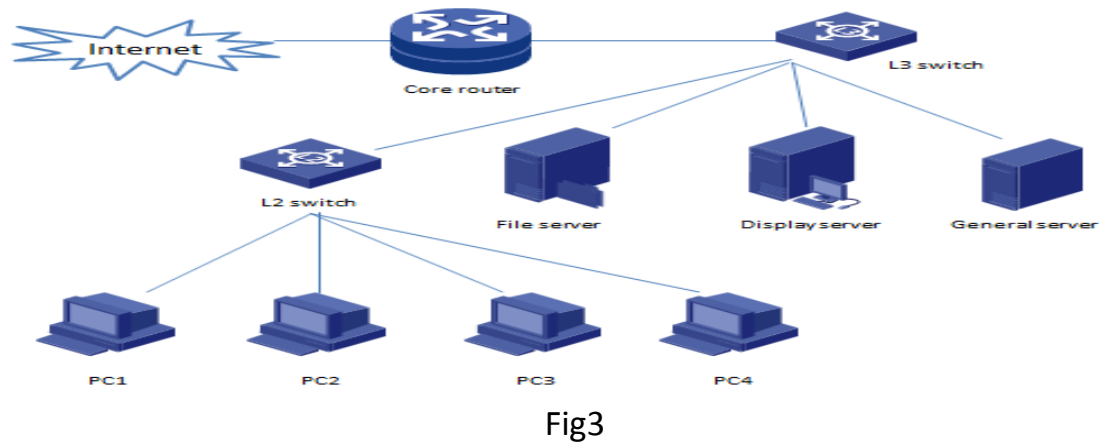

\section{Summary}

The rapid development computer network more reflected in the improved performance network hardware, computer software user interface earth-shaking changes and software applications methods of operation greatly simplify humane. Through the entire computer network technology training also enables students to understand the common computer and computer network failure, and master the ways to remove. At the same time allows students grasp the basic network software application knowledge to understand today state-of-the-art computer network hardware and mastery of the basic management hardware, so that students can gradually accept new things in teaching learning equipment, to understand new knowledge, with ability to learn.

\section{References}

[1] Wang Runyun. Basic computer courses teaching reform and practice [J]. Journal of education development research, 2003, (2).

[2]Teng Bu Hui. Analyses the university computer foundation course teaching ideas and methods [J]. Journal of lianyungang chemical junior high school

[3]Cui Xuanhui Wang Zhixiang. Basic computer course teaching reform and deepen the reform of thinking [J]. Journal of chongqing industrial college, 2007

[4]Luo Fuwu.Discuss about the training goal of higher engineering education. Tsinghua university education research.

[5]Chen Xiaodong. The whole of the theory of vocational education teaching reform forward. 